\title{
Die Spectral Illumination (SIL) Methode: Eine Versuchsreihe
}

\author{
Theo Gabloffsky, Jannika Lossner, Julia Richardt, Ralf Salomon \\ Universität Rostock \\ Institut für Angewandte Mikroelektronik und Datentechnik \\ 18051 Rostock \\ \{theo.gabloffsky, jannika.lossner, julia.richardt, \\ ralf.salomon\}@uni-rostock.de
}

\begin{abstract}
Zusammenfassung. Eine klassische Problemstellung in der heutigen Technik ist die computergestützte Erkennung von Farben. So ist es beispielsweise für manche Tierexperimente interessant, Bewegungsmuster von Tieren automatisch zu erkennen und auszuwerten. Ein Lösungsansatz besteht darin, die Tiere mit mehreren Farbmarkern zu versehen und zu filmen, um aus den Bewegungen der Farbpunkte abzuleiten, wie sich die Tiere bewegen. Gerade in Versuchsumgebungen mit wechselender oder abgeschwächter Beleuchtung kann die Unterscheidung der Farben jedoch eine Herausforderung sein. Dieser Beitrag schlägt vor, das Szenario mit sehr schnell wechselnden schmalbandigen Spektralfarben zu beleuchten. Je nach Kombination aus Wellenlänge und Farbmarker entstehen so unterschiedliche Bilder ein und der selben Situation, welche aufgrund der unterschiedlichen Farbeindrücke der Bilder für eine bessere Auswertung der Situation genutzt werden können.
\end{abstract}

\section{Einführung}

Neue Medikamente werden nach ihrer Entwicklung nicht einfach für Menschen zugelassen. Um ihre Wirksamkeit nachzuweisen und unerwünschte Nebeneffekte auszuschließen werden sie im Rahmen vorklinischer Studien an Tieren intensiv getestet. Des Weiteren gibt es Krankheiten, die nur an Menschen auftreten. Für die Entwicklung geeigneter Therapien ist es in diesen Fällen notwendig, entsprechende tierische Modelle zu entwickeln.

Morbus Parkinson ist ein gutes Beispiel für obige Ausführungen. Diese Krankheit ist nur bei Menschen beobachtbar. Um geeignete Modelle zu haben, werden Ratten einseitig läsiert und anschließend therapiert. Der Erfolg beider Maßnahmen führt zu einem asymmetrischen Verhalten des Versuchsobjektes und kann anhand signifikanter Rotationsbewegungen beobachtet werden [ $\left.\mathrm{VAE}^{+} 16\right]$.

Für die Beobachtung der Rotationsbewegungen werden die Ratten in einen größeren, blumentopfartigen Trog gesetzt. Zusätzlich wird den Ratten nach dem Stand der Technik $\left[\mathrm{VAE}^{+} 16\right]$ ein kleiner Brustgurt angelegt, der über einen schmalen Draht mit einem Rotationszähler verbunden ist. Dieser Versuchaufbau ist grundsätzlich funktionsfähig, hat aber eine Reihe von Nachteilen. Einer 
dieser Nachteile ist, dass die Rotationszähler am Ende des Versuches nur Nettozahlen hinsichtlich der beiden Drehrichtungen liefern; ein zeitlicher Verlauf ist nicht verfügbar. Des Weiteren schränkt der gängige Versuchsaufbau die Bewegungsfreiheit sowie den Aktionsraum der Ratten ein.

Zur Beseitigung obiger Nachteile wurde in der Vergangenheit ein erstes berührungsloses System entwickelt [JZHS16]. Bei diesem Ansatz werden Farbmarker auf das Rattenfell aufgetragen. Während des Versuches wird die Ratte mittels einer überkopf montierter Kamera beobachtet. Eine entsprechende Software wertet die gelieferten Bilder aus und stellt den abgeleiteten Rotationsverlauf zeitlich aufgelöst zur Verfügung.

Obwohl prinzipiell funktionsfähig, haftet dem beschriebenen Ansatz der Nachteil an, dass die einzelnen Farben unter der gegebenen Beleuchtung nicht gut voneinander unterscheidbar sind. Die von der Kamera gelieferten RGB Werte unterscheiden sich je nach Situation nur marginal voneinander. Als Konsequenz kann die Verwendete Bilderkennungssoftware keine zuverlässigen Rotationsdaten liefern. Die Beleuchtung des Versuchsumfeldes muss aufgrund der Lichtempfindlichkeit der Ratten relativ dunkel gehalten werden. Eine Verstärkung des Lichtes könnte zu einem verändertem Verhalten der Ratte führen.

Eine Möglichkeit, die Farberkennung zu vereinfachen, wäre, mehrere Kameras mit vorgeschalteten Filtern zu verwenden. Dies ist jedoch aufwändig und aufgrund eines hohen Platzbedarfes im Versuchsumfeld nicht möglich. Dieser Beitrag bespricht einen alternativen Ansatz für eine verbesserte Farbdifferenzierung.

Dieser Ansatz wird im Abschnitt 3 vorgestellt und mit einem prinzipiellen Vorgehen beschrieben. Die Leistungsfähigkeit des Ansatzes wurde in einem Laborexperiment evaluiert. Dieses wird in Abschnitt 4 beschrieben und soll die Möglichkeit bieten, das Experiment nachzustellen. Die Ergebnisse sind in Abschnitt 5 dargestellt und werden im nachfolgenden Abschnitt 6 besprochen.

\section{Stand der Technik}

Die in [JZHS16] angewendete Methodik der Farberkennung ist eine Form der sogenannten Blob Detection. Diese zielt darauf ab, eine bestimmte Region innerhalb eines Motives über bestimmte farbliche Eigenschaften vom Hintergrund zu unterscheiden. Sollten sich diese Eigenschaften zu sehr ähneln, ist eine Unterscheidung nicht mehr möglich. Konkret bedeutet das, dass bei jedem Pixel innerhalb eines Bildes untersucht wird, ob sein Farbwert einem gesuchten Farbwert entspricht. Aus den gefundenen Pixeln wird dann der Schwerpunkt berechnet. Im Idealfall ist dieser dann auch der Mittelpunkt des gesuchten Farbkreises. Ein alternativer Ansatz zur Blob Detection stellt die Methode des Histogram Comparison dar. Bei dieser Methode wird innerhalb eines Bildes nach einem Bildausschnitt oder Fenster gesucht, dessen farbliches Histogram dem eines Referenz-Histogrammes entspricht. Weiterführende Methoden, wie beispielsweise in [PHVG02] und [DKFvdW14] beschrieben, ermöglichen eine ressourcenspa- 
rende, flexible und robuste Alternative zur Blob Detection. Anwendungsbeispiele für diese Techniken sind das Objekt-Tracking von beispielsweise Personen oder Autos. Die Idee zur multispektralen Beleuchtung eines Motives wurde grundsätzlich bereits in [CYBE08] und [PLGN07] beschrieben. Die Idee ist dabei, dass ein Motiv anstelle von einer einzigen breitbandigen Lichtquelle mit mehreren, schmalbandigen Lichtquellen beleuchtet wird. Dabei wird versucht, aus den einzelnen spektralen Antworten mehr Informationen zu gewinnen, als es mit einer breitbandigen Beleuchtungsquelle möglich wäre.

\section{Die Spectral Illumination (SIL) Methode}

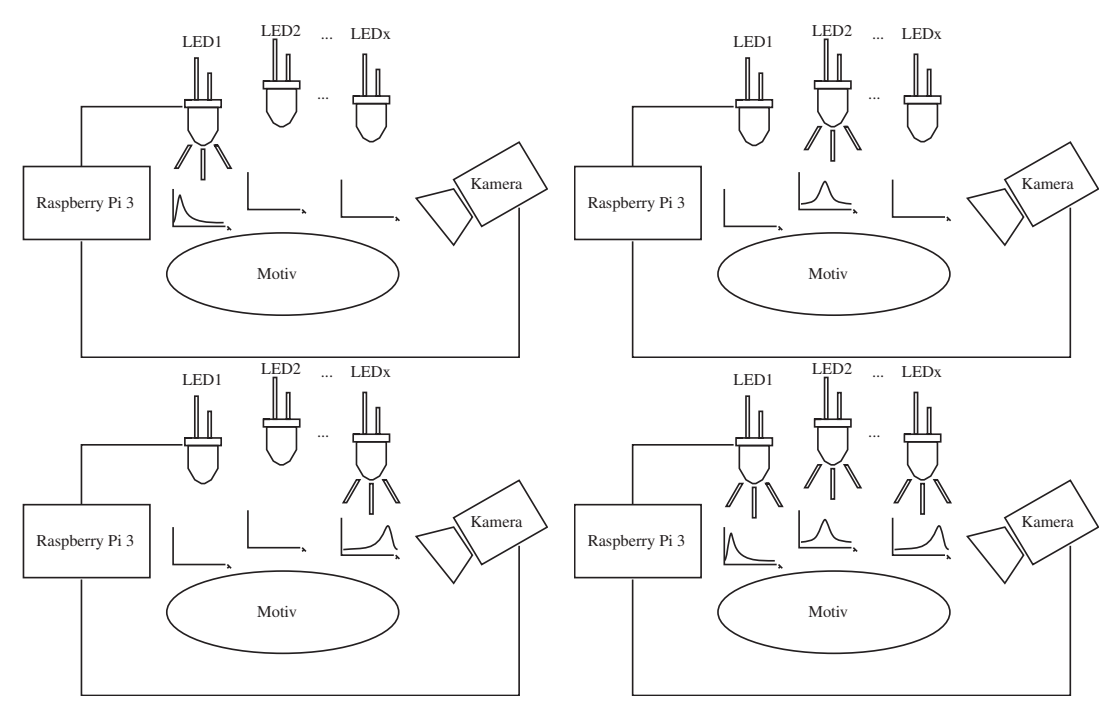

Abb. 1. Schematischer Versuchsaufbau und Ablauf der SIL-Methode

Für die multispektrale Beleuchtung wird eine Beleuchtungseinheit benötigt, die in der Lage ist, mit unterschiedlichen Wellenlängen schmalbandig zu beleuchten. Synchron zur Beleuchtung des Motives nimmt eine Kamera Bilder auf, die anschließend ausgewertet werden. In Abbildung 1 ist ein schematischer Aufbau und ein beispielhafter Ablauf skizziert.

Die Idee hinter der Methode ist, dass sich durch die unterschiedliche spektrale Beleuchtung bestimmte Farbeigenschaften des Rattenfelles und der verwendeten Farben ausnutzen lassen, um die Farbdistanzen zweier Punkte zu vergrößern. Je nach Beleuchtung wäre somit die Unterscheidung von zwei unterschiedlich farbigen Punkten einfacher. 


\section{Praktische Versuche}

Die Leistungsfähigkeit der SIL-Methode wurde in einigen Labormessungen evaluiert. Dieser Abschnitt beschreibt den Versuchsaufbau, das Vorgehen und alle relevanten Parameter um die Messungen nachzustellen.

Versuchsaufbau: Der Versuchsaufbau bestand aus einem Raspberry Pi Model 3, der Raspberry Pi Kamera Version 2, einer LED-Bank die als konfigurierbare Lichtquelle arbeitet, sowie aus den Testmotiven. Der Raspberry Pi diente dabei als Steuergerät für den gesamten Aufnahmeprozess. Er kontrollierte die Lichtquelle und veranlasste die Kamera zur Bildaufnahme. Die Bildgröße betrug 1024 $\times 768$ Pixel in einem RGB8 Bildformat. Sämtliche Aufnahmen wurden innerhalb einer Dunkelkammer durchgeführt.

Lichtquelle: Die Lichtquelle besteht aus drei LED-Typen à vier LEDs, mit den Wellenlängen 620-625 nm, 520-525 nm und 459-462 nm. Von diesen LED-Typen sind jeweils vier zusammen auf verteilten Platinen untergebracht. Diese Platinen sind weiterhin mit einer Logikplatine verbunden, über die der Raspberry Pi 3 die unterschiedlichen LEDs ansprechen kann. Die Aufteilung der LEDs auf unterschiedliche Platinen ermöglicht eine genaue Ausrichtung auf das Motiv.

Versuchsreihen: Aufgrund der in der Einleitung beschriebenen Problematik der Farberkennung auf einem Rattenfell, wurden die Versuchsreihen ebenfalls auf einem Rattenfell durchgeführt. Als Farbgeber wurden Tiermarkierungsstifte der Firma Raidex in unterschiedlichsten Farben verwendet. [Rai18]

Kamera: Das Raspberry Pi Kameramodul in der Version 2 verwendet einen Sony IMX219 Bildsensor. Dieser bietet eine Auflösung von 8 Megapixel und basiert auf der Active-Pixel-Technologie. Weiterhin ist dem Bildsensor ein RGBFiltermosaik vorgeschaltet, welches dem Bildsensor die Farberkennung ermöglicht [IMX17]. Der genaue Aufbau des Mosaiks sowie die Verhältnisse der grünen, roten und blauen Farbfilter zueinander sind nicht bekannt. Diese Verteilung hat jedoch einen signifikanten Einfluss auf die Farberkennung der Kamera. Um diese Unbekannte auszugleichen, wurden Referenzeinstellungen getroffen, die zum einen die unterschiedliche Aufnahmecharakteristik der Kamera und zum anderen auch die unterschiedliche Abstrahlleistungen der LEDs kompensieren soll. Diese Einstellungen beinhalten eine Verstärkung der rot- und blau-Töne in dem Maß, dass bei einer Bildaufnahme mit angeschaltener Lichtquelle ein gewählter Punkt auf dem weißen Fell die Farbkanäle in gleicher Stärke anspricht. Die gesetzten Parameter können der Tabelle 1 entnommen werden.

\section{Bildverarbeitung:}

Die Weiterverarbeitung der Bilddaten beinhaltete das Anwenden eines GaussFilters sowie eines Normalisierungsfilters. Der Gauss-Filter glättet strukturelle Gegebenheiten des Fells und sorgt für eine homogene Farbverteilung. Er wird innerhalb der Bildverarbeitung durch eine zweidimensionale Gauss-Matrix mit einer bestimmten Größe charakterisiert. Diese Matrix $h$ berechnet sich aus den 
Tabelle 1. Kameraeinstellungen

\begin{tabular}{ll}
\hline Parameter & Wert \\
\hline rot Verstärkung & 2,7 \\
blau Verstärkung & 1,5 \\
Belichtungszeit & 5000 us \\
ISO-Wert & 350 \\
\hline
\end{tabular}

Matrix-Koordinaten und einer Standardabweichung $\sigma$ nach folgender Formel.

$$
h(x, y)=\frac{1}{2 \pi \sigma^{2}} e^{-\frac{x^{2}+y^{2}}{2 \sigma^{2}}}
$$

Der für die praktischen Versuche verwendete Gauss-Filter hat eine Matrix-Große von $7 \times 7$ und verwendet eine Standardabweichung $\sigma=15$. Diese Gauss-Matrix wird dann mit jedem Pixel $\left(P_{\text {alt }}\right)$ des ursprünglichen Bildes nach folgender Formel gefaltet.

$$
P_{n e u}(x, y)=\sum_{i=1}^{n} \sum_{j=1}^{n} P_{a l t}(x-i+a, y-j+a) k(i, j)
$$

Das Ergebnis ist eine weichgezeichnete Version des orginalen Bildes. Je nach Parameterwahl verändert sich der Grad der Verschmierung. Der Normalisierungsfilter setzt alle Bilder auf eine gleiche, durchschnittliche Helligkeit. Als Referenzhelligkeit wurde dabei die durchschnittliche Helligkeit des hellsten Bildes aus der Messreihe verwendet. Für die Berechnung der Helligkeit eines jeden Pixels wurden die vorhandenen RGB-Daten in den HSV-Farbraum umgewandelt, mit einem entsprechend Offset auf den V-Parameter belegt und wieder zurück in den RGB-Raum gewandelt. Eine weitere Software ermittelt die Farbdistanzen aus ausgewählten Bereichen der Bilder nach folgender Formel:

$$
d=\sqrt{\left(R_{p 1}-R_{p 2}\right)^{2}+\left(G_{p 1}-G_{p 2}\right)^{2}+\left(B_{p 1}-B_{p 2}\right)^{2}}
$$

\section{$5 \quad$ Ergebnisse}

In diesem Abschnitt werden ausgewählte Farbdistanzen für die LED-Farben Rot (R), Grün (G) und Blau (B) vorgestellt. Als Farbpunkte wurden Farben in Rot, Grün und Blau gewählt. Ein weiterer Referenzpunkt ist ein Punkt auf dem Rattenfell, der im folgenden als ref abgekürzt wird. Als Beispiel brachte die Beleuchtung eines roten Farbklecks mit einer roten Beleuchtung (Tabelle 2) nur eine Farbdistanz von 27,20 gegenüber dem ausgewähltem Referenzpunkt. Die Farben des roten Flecks und des Referenzpunktes sind sich also ähnlich. Wird dieser rote Fleck hingegen mit allen LEDs (also R, G und B) beleuchtet, zeigt 
sich eine Farbdistanz von 223, 33. Die beiden Punkte sind klarer voneinander zu unterscheiden.

Tabelle 2. Farbdistanzen ohne Filterung

\begin{tabular}{llllllll}
\hline Farbklecks/Beleuchtung & $\mathrm{R}$ & $\mathrm{G}$ & $\mathrm{B}$ & $\mathrm{RG}$ & $\mathrm{RB}$ & $\mathrm{GB}$ & $\mathrm{RGB}$ \\
\hline ref/rot & 27,20 & 172,46 & 167,16 & 170,25 & 162,79 & 223,12 & 223,33 \\
ref/grün & 168,44 & 102,26 & 146,14 & 187,58 & 218,83 & 165,65 & 221,79 \\
ref/blau & 176,42 & 144,40 & 113,11 & 213,01 & 202,65 & 175,39 & 236,69 \\
rot/grün & 142,36 & 82,22 & 22,49 & 184,96 & 139,67 & 89,56 & 183,12 \\
rot/blau & 150,34 & 53,82 & 54,67 & 168,03 & 151,69 & 80,48 & 166,75 \\
blau/grün & 8,00 & 43,60 & 33,06 & 48,08 & 33,30 & 63,09 & 61,54 \\
\hline
\end{tabular}

Tabelle 3. Farbdistanzen nach Gauss-Filterung

\begin{tabular}{llllllll}
\hline Farbklecks/Beleuchtung $\mathrm{R}$ & $\mathrm{G}$ & $\mathrm{B}$ & $\mathrm{RG}$ & $\mathrm{RB}$ & $\mathrm{GB}$ & $\mathrm{RGB}$ \\
\hline ref/rot & 28,46 & 171,25 & 167,13 & 171,71 & 160,46 & 222,18 & 222,79 \\
ref/grün & 167,39 & 102,59 & 144,16 & 190,80 & 217,43 & 164,61 & 232,55 \\
ref/blau & 176,42 & 148,60 & 112,14 & 214,67 & 204,03 & 181,41 & 241,93 \\
rot/grün & 140,30 & 79,67 & 23,77 & 185,74 & 140,52 & 91,01 & 188,89 \\
rot/blau & 149,35 & 49,43 & 55,48 & 166,89 & 153,50 & 80,11 & 171,85 \\
blau/grün & 9,06 & 47,36 & 32,08 & 48,18 & 31,64 & 61,43 & 60,67 \\
\hline
\end{tabular}

Tabelle 4. Farbdistanzen nach Helligkeits-Normalisierung

\begin{tabular}{llllllll}
\hline Farbklecks/Beleuchtung & $\mathrm{R}$ & $\mathrm{G}$ & $\mathrm{B}$ & $\mathrm{RG}$ & $\mathrm{RB}$ & $\mathrm{GB}$ & $\mathrm{RGB}$ \\
\hline ref/rot & 27,51 & 180,58 & 167,10 & 178,20 & 160,82 & 223,23 & 225,33 \\
ref/grün & 168,53 & 102,42 & 146,14 & 192,94 & 218,07 & 165,89 & 221,79 \\
ref/blau & 176,51 & 144,37 & 113,11 & 216,03 & 200,96 & 176,22 & 236,69 \\
rot/grün & 142,54 & 92,66 & 22,14 & 193,90 & 138,41 & 92,92 & 183,12 \\
rot/blau & 150,51 & 64,20 & 54,49 & 173,58 & 149,12 & 83,12 & 166,75 \\
blau/grün & 8,00 & 44,02 & 33,06 & 48,26 & 33,20 & 65,28 & 61,54 \\
\hline
\end{tabular}


Tabelle 5. Farbdistanzen nach Gauss-Filterung und Helligkeitsnormalisierung

\begin{tabular}{llllllll}
\hline Farbklecks/Beleuchtung & $\mathrm{R}$ & $\mathrm{G}$ & $\mathrm{B}$ & $\mathrm{RG}$ & $\mathrm{RB}$ & $\mathrm{GB}$ & $\mathrm{RGB}$ \\
\hline ref/rot & 28.72 & 178,17 & 167,09 & 179,77 & 158,49 & 225.32 & 225,62 \\
ref/grün & 167,47 & 102,77 & 144,16 & 196,17 & 216,66 & 164.84 & 233,46 \\
ref/blau & 176,51 & 148,62 & 112,14 & 217,69 & 202,34 & 184.23 & 243,21 \\
rot/grün & 140,49 & 89,25 & 23,56 & 194,63 & 139,27 & 94,39 & 192,04 \\
rot/blau & 149,54 & 59,21 & 55,34 & 172,51 & 150,96 & 82,95 & 174,40 \\
blau/grün & 9,06 & 47,55 & 32,08 & 48,41 & 31,46 & 63,66 & 62,90 \\
\hline
\end{tabular}

\section{Diskussion}

Ziel der vorliegenden Messungen war es, zu untersuchen, inwiefern eine schmalbandige spektrale Beleuchtung eines Motives die Farbdifferenzierung zweier Farbpunkte verbessern könnte. Die Ergebnisse zeigen, dass die Farbdistanz zwischen zwei Farben unter einer spektralen Beleuchtung weniger groß ausfällt als unter einer breitbandigen Beleuchtung. Als Beispiel dafür seien die Farbabstände aus Tabelle 2 genannt. Hier zeigte die Beleuchtung eines blauen Farbkleckses mit einer roten Beleuchtung eine Farbdistanz von 176.42 gegenüber dem gewähltem Referenzpunkt. Bei voller Beleuchtung konnte eine Farbdistanz von 236,69 ermittelt werden. Es zeigt sich also, dass sich die gewählte Farbe bei voller Beleuchtung stärker von dem Referenzpunkt abhebt als bei einer rein spektralen Beleuchtung. Auch die Anwendung von unterschiedlichen Filtern, wie in Abschnitt 4 beschrieben, erbrachte keine Erhöhung der Farbdistanz. So zeigte sich zwar, dass durch die Anwendung eines Gaussfilters die Struktur des Rattenfells geglättet werden konnte, was jedoch keinen Vorteil hinsichtlich der Farbdistanz erbrachte. Die Erklärung für eine verringerte Farbdistanz bei einer rein spektralen Beleuchtung lautet wie folgt: Der RGB-Farbraum wird durch einen dreidimensionalen Raum, bei dem die Achsen den Grundfarben Rot, Grün und Blau entsprechen, beschrieben. Für die Wahrnehmung der Farbe eines Objektes ist nicht nur die Farbe selbst entscheidend, sondern auch mit welchem Licht diese Farbe beleuchtet wird. So kann der Farbraum nur dann voll ausgenutzt werden, wenn gleichermaßen mit rotem, grünem und blauem Licht beleuchtet wird. Wird ein Anteil der Farbe in der Beleuchtung komplett weggelassen, so verringert sich der dreidimensionale Farbraum zu einem zweidimensionalem Raum, was die Farbdistanzen verkleinert oder bestenfalls gleich lässt. Eine Ausnahme können dabei fluoriszierenden Farben bilden, die das eingestrahlte Licht in eine höhere Wellenlänge umwandeln und damit die Farbdistanz erhöhen. Weitere Untersuchungen sollten darauf abzielen, fluoriszierende Farben als Farbpunkte zu verwenden um die Farbdistanzen zu erhöhen.

\section{Zusammenfassung}

Die Aufgabenstellung dieses Beitrages war die Verbesserung der Farbdifferenzierungen im Umfeld medizinischer Tierversuche. Zur Lösung der Problemstellung 
hat der vorliegende Beitrag die Methode der spektralen Beleuchtung (SIL) mit einem Beispielaufbau und Beispielvorgehen vorgestellt. Um die Leistungsfähigkeit der SIL-Methode zu evaluieren, wurden mehrere Messaufnahmen mit unterschiedlichen Farben und Beleuchtungen vorgenommen. Um äußere Einflüsse und Ungenauigkeiten in der Messaufnahme zu verringern, wurden die Bilder mit Hilfe von digitalen Filtern nachbearbeitet. Anschließend konnten die aufgenommenen Ergebnisse ausgewertet und die Leistungsfähigkeit der SIL-Methode bewertet werden. Die Ergebnisse sind negativ ausgefallen und es konnte keine verbesserte Farbdifferenzierung auf Grundlage der Farbdistanz erzielt werden. Die nächsten Arbeitsschritte werden weitere Untersuchung sein, die als Farbstoff fluoriszierende Farben und unterschiedliche Kameras verwenden werden. Diese zeigten in ersten Versuchen positive Ergebnisse, was die Leistungsfähigkeit der Methode nachweisen könnte.

\section{Danksagung}

Wir bedanken uns an dieser Stelle bei Herrn Dr. Alexander Hawlischka und Herrn Prof. Wree von der Universität Rostock für die interessante Aufgabenstellung und für die Bereitstellung von Unterlagen und Materialien. Weiterhin danken wir Prof. Nils Damaschke für nützliche Hinweise und Tipps bei der Umsetzung der Messungen.

\section{Literatur}

[CYBE08] Cui Chi, Hyunjin Yoo und Moshe Ben-Ezra. Multi-Spectral Imaging by Optimized Wide Band Illumination. International Journal of Computer Vision, 86(2):140, Nov 2008.

[DKFvdW14] M. Danelljan, F. S. Khan, M. Felsberg und J. v. d. Weijer. Adaptive Color Attributes for Real-Time Visual Tracking. In 2014 IEEE Conference on Computer Vision and Pattern Recognition, Seiten 1090-1097, June 2014.

[IMX17] IMX219PQ Product Brief V1.0. https://www.sony-semicon.co.jp/ products_en/IS/sensor1/img/products/ProductBrief_IMX219PQ_ 20160425.pdf, 2017.

[JZHS16] Ralf Joost, Daniel Ziese, Alexander Hawlitschka und Ralf Salomon. Mouse Pi: A Platform for Monitoring In-Situ experiments. In Tagungsband des 5. Jahreskolloquiums Bildverarbeitung in der Automation (BVAu 2016), Seiten 1-5, 2016.

[PHVG02] P. Pérez, C. Hue, J. Vermaak und M. Gangnet. Color-Based Probabilistic Tracking. In Anders Heyden, Gunnar Sparr, Mads Nielsen und Peter Johansen, Hrsg., Computer Vision - ECCV 2002, Seiten 661-675, Berlin, Heidelberg, 2002. Springer Berlin Heidelberg.

[PLGN07] J. Park, M. Lee, M. D. Grossberg und S. K. Nayar. Multispectral Imaging Using Multiplexed Illumination. In 2007 IEEE 11th International Conference on Computer Vision, Seiten 1-8, Oct 2007.

[Rai18] Raidex Viehzeichenstifte. https://www.raidex.de/produkte/ viehzeichenstifte/, 2018. 
[VAE ${ }^{+}$16] Antipova V*, Hawlitschka A*, Mix E, Schmitt O, Dräger O, Dräger D, Benecke $\mathrm{R}$ und Wree A. Behavioral and structural effects of unilateral intrastriatal injections of botulinum neurotoxin a in the rat model of Parkinson disease. In Tagungsband des 5. Jahreskolloquiums Bildverarbeitung in der Automation (BVAu 2016), 2016.

Open Access Dieses Kapitel wird unter der Creative Commons Namensnennung 4.0 International Lizenz (http://creativecommons.org/licenses/by/4.0/deed.de) veröffentlicht, welche die Nutzung, Vervielfältigung, Bearbeitung, Verbreitung und Wiedergabe in jeglichem Medium und Format erlaubt, sofern Sie den/die ursprünglichen Autor(en) und die Quelle ordnungsgemäß nennen, einen Link zur Creative Commons Lizenz beifügen und angeben, ob Änderungen vorgenommen wurden.

Die in diesem Kapitel enthaltenen Bilder und sonstiges Drittmaterial unterliegen ebenfalls der genannten Creative Commons Lizenz, sofern sich aus der Abbildungslegende nichts anderes ergibt. Sofern das betreffende Material nicht unter der genannten Creative Commons Lizenz steht und die betreffende Handlung nicht nach gesetzlichen Vorschriften erlaubt ist, ist für die oben aufgeführten Weiterverwendungen des Materials die Einwilligung des jeweiligen Rechteinhabers einzuholen. 